\title{
Tethered Particle Motion as a Diagnostic of DNA Tether Length
}

\author{
Philip C. Nelson, ${ }^{\dagger}$ Chiara Zurla, ${ }^{\ddagger}$ Doriano Brogioli,,${ }^{\ddagger}$ John F. Beausang, ${ }^{\dagger}$ Laura Finzi,,${ }^{\S}$ and \\ David Dunlap*,ll
}

Department of Physics and Astronomy, University of Pennsylvania, Philadelphia, Pennsylvania 19104, Department of Biology, University of Milan, 20133 Milan, Italy, and Departments of Physics and Cell Biology, Emory University, Atlanta, Georgia 30322

Received: May 18, 2006; In Final Form: June 29, 2006

\begin{abstract}
The tethered particle motion (TPM) technique involves an analysis of the Brownian motion of a bead tethered to a slide by a single DNA molecule. We describe an improved experimental protocol with which to form the tethers, an algorithm for analyzing bead motion visualized using differential interference contrast microscopy, and a physical model with which we have successfully simulated such DNA tethers. Both experiment and theory show that the statistics of the bead motion are quite different from those of a free semiflexible polymer. Our experimental data for chain extension versus tether length fit our model over a range of tether lengths from 109 to 3477 base pairs, using a value for the DNA persistence length that is consistent with those obtained under similar solution conditions by other methods. Moreover, we present the first experimental determination of the full probability distribution function of bead displacements and find excellent agreement with our theoretical prediction. Our results show that TPM is a useful tool for monitoring large conformational changes such as DNA looping.
\end{abstract}

\section{Introduction}

Many different techniques have been developed to study the mechanical properties of single DNA molecules. Some of them consist of tethering a bead to a surface or to another bead through a DNA molecule. Then, forces may be applied to the beads, by using optical traps, ${ }^{1,2}$ magnets, ${ }^{3,4}$ or some combination of fluid flow, osmotic force, and electrostatic repulsion. ${ }^{5}$ In the simplest case, the so-called tethered particle motion (TPM) technique, a bead is tethered to the surface of a slide by a single DNA molecule and undergoes Brownian motion in the absence of externally applied force (Figure 1). Lateral displacements are observed through an optical microscope, to evaluate the amplitude of motion of the bead that depends on the DNA length. Optical techniques including differential interference contrast and evanescent wave microscopy have been used to monitor the motion of the beads..$^{6,7}$

Besides its simplicity, TPM has some advantages over other similar techniques. In particular, it is suited for the study of short molecules (from 100 to several thousand base pairs (bp) long) undergoing length or elasticity variations. ${ }^{6,8}$ Moreover, TPM allows the study of proteins acting on torsionally relaxed molecules not subjected to externally applied tension., ${ }^{5,79-12}$ Various algorithms have been employed to evaluate the amplitude of motion, and two experimental calibration curves have been measured in order to relate the amplitude of motion of the bead to the length of the DNA molecule. ${ }^{8,13}$ Similarly, we describe an experimental protocol and an algorithm for quantitative data analysis. Using DNA molecules of different lengths, we measured the amplitudes of Brownian motion of

* To whom correspondence should be addressed. Present address: Department of Cell Biology, Emory University School of Medicine, 615 Michael Street, Atlanta, GA 30322. Phone: 404-727-7729. Fax: 404-7276256. E-mail: ddunlap@emory.edu.

University of Pennsylvania.

$\doteqdot$ University of Milan.

$\S$ Department of Physics, Emory University.

"Department of Cell Biology, Emory University.

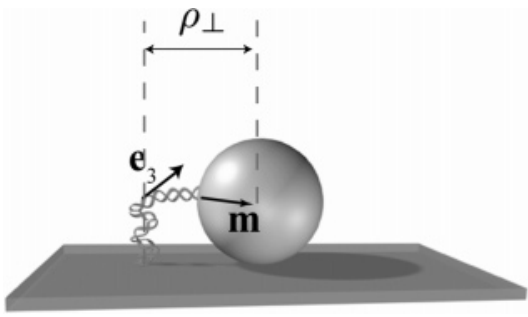

Figure 1. In the tethered particle motion technique, a DNA molecule flexibly links a bead to a surface. The motion of the bead is passively observed and automatically tracked. For the position of the bead in each video frame, the projected separation vector from the anchor point, $\rho_{\perp}$, is determined. The simulation described below attributes a tangent vector, $\mathbf{e}_{3}$, to each point along the DNA chain, and a final vector, $\mathbf{m}$, from the end of the DNA to the center of the bead. As suggested in the figure, steric exclusion of the bead from the surface significantly alters the DNA chain statistics.

beads to construct a calibration curve, and compare to those previously reported.

We also describe and validate a physical model with which to simulate TPM experiments. This model goes beyond previously reported scaling analysis ${ }^{13}$ and, unlike other work, ${ }^{14}$ is specifically adapted to modern single-particle-tracking methods. It is equivalent to a model described by Segall and collaborators, ${ }^{15}$ but here, we give the first comparison to experimental data. Fitting the single-parameter model to the measured motions of tethered beads produced a persistence length for DNA that is consistent with previous experimental evaluations in similar buffer solutions. Then, using Monte Carlo simulation of the physical model, we predicted the probability distributions of Brownian motion for tethered beads and found good agreement with our experimental data.

\section{Sample Preparation}

Some DNA fragments were obtained by polymerase chain reaction (PCR) amplification from plasmid templates. Among 
these, certain PCR fragments were labeled during amplification using biotin- and digoxigenin-modified primers (MWG Biotech AG, Ebersberg, Germany). Others, amplified using unmodified primers and purified plasmids cleaved with restriction endonucleases (Qiagen GmbH, Hilden, Germany), were labeled on 3'recessed ends filled with bio-dUTP and dig-dUTP (Roche Diagnostics GMBH, Mannheim, Germany) by Klenow DNA polymerase (Fermentas UAB, Vilnius, Lithuania).

Preparation of the Flow Chamber for Tethered Particle Motion. Details about the flow chambers for TPM measurements have been reported. ${ }^{16,17}$ A flow chamber was incubated overnight at $4{ }^{\circ} \mathrm{C}$ with $40 \mu \mathrm{g} / \mathrm{mL}$ biotin-labeled BSA (Sigma, St. Louis, MO). After washing the flow chamber with $800 \mu \mathrm{L}$ of buffer (10 mM Tris- $\mathrm{HCl}(\mathrm{pH} 7.4), 200 \mathrm{mM} \mathrm{KCl}, 5 \%$ dimethyl sulfoxide (DMSO), $0.1 \mathrm{mM}$ ethylenediaminetetraacetic acid (EDTA), $0.2 \mathrm{mM}$ dithiothreitol (DTT), and $0.1 \mathrm{mg} / \mathrm{mL}$ $\alpha$-casein), the chamber was incubated for $2 \mathrm{~h}$ with $50 \mu \mathrm{g} / \mathrm{mL}$ streptavidin (Sigma, St. Louis, MO). Separately, DNA was incubated for $2 \mathrm{~h}$ with an excess of antidigoxigenin-coated beads (480 nm in diameter, Indicia Diagnostics, Oullins, France) and suspended in buffer lacking DTT and DMSO. After washing the chamber with $800 \mu \mathrm{L}$ of buffer, biotin- and digoxigeninlabeled DNA was introduced and incubated for $1 \mathrm{~h}$. Unattached beads were then flushed from the chamber with buffer.

Tethered Particle Motion (TPM) Measurement. Within each field of view, four to six visibly mobile beads were observed using differential interference contrast (DIC) microscopy. The microscope (Leica DM LB-100, Leica Microsystems, Wetzlar, Germany) was equipped with an oil-immersion objective $(\mathrm{N}$-Plan $100 \times 1.4)$ and a CV-A60 CCD camera (JAI, Copenhagen, Denmark). The video sequence was digitized with an IMAQ PCI-1409 frame grabber (National Instruments, Austin, TX) and analyzed using a custom Lab View (National Instruments, Austin, TX) routine. The exposure time of the CCD camera was minimized to avoid blurring that otherwise reduced the apparent amplitude of the motion of the bead. ${ }^{18}$ The exposure had to be much shorter than the time, $t_{\mathrm{b}}$, required by the bead to traverse its range of motion. A rough estimate is given by $t_{\mathrm{b}}$ $=\sigma^{2} / D$, where $\sigma$ is the range of motion and $D \approx 1000 \mathrm{~nm}^{2} / \mathrm{ms}$ is the diffusion coefficient of the bead obtained using the Stokes-Einstein formula. Typical values of $t_{\mathrm{b}}$ are about $50 \mathrm{~ms}$, and indeed, we found that $40 \mathrm{~ms}$ exposures reduced the apparent amplitude of motion by about $20 \%$. Instead, we used $1 \mathrm{~ms}$ exposures, which are safely lower than $t_{\mathrm{b}}$.

Standard PAL video cameras use an interlaced format: the even lines of each image are exposed $20 \mathrm{~ms}$ after the odd lines. To avoid blur from this $20 \mathrm{~ms}$ offset, we analyzed the even and odd fields as independent sequences. This procedure effectively doubled the pixel spacing in the vertical direction (from 64 to $128 \mathrm{~nm}$ in the field of view) but did not significantly impair our determination of bead positions.

\section{Data Acquisition}

In a single field of view, the operator manually selected four to six beads exhibiting the largest amplitudes of Brownian motion. Beads viewed with DIC appear as juxtaposed bright and dark semicircles on a featureless gray background, which simplified tracking. After selection of a small region containing the bead from an initial frame, the position of the bead was automatically determined throughout the sequence with the following algorithm: (1) Establish the maximum (minimum) and average intensities. (2) Define a threshold at $30 \%$ of the maximum (minimum) with respect to the average. (3) Select the contiguous pixels with intensity above (below) the threshold value, starting from the highest level. (4) Establish the center

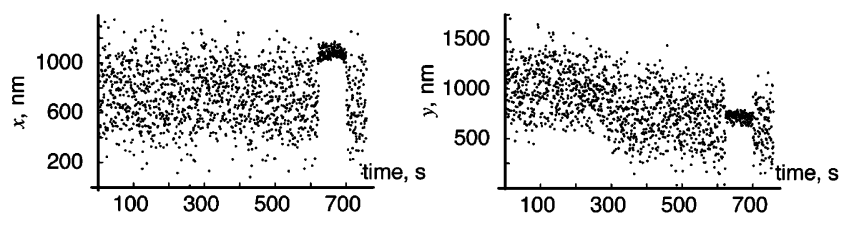

Figure 2. Time series for a $3477 \mathrm{bp}$ long tether, showing a transient sticking event and also instrumental drift. The data have been culled for visualization; one out of every 20 points is shown.

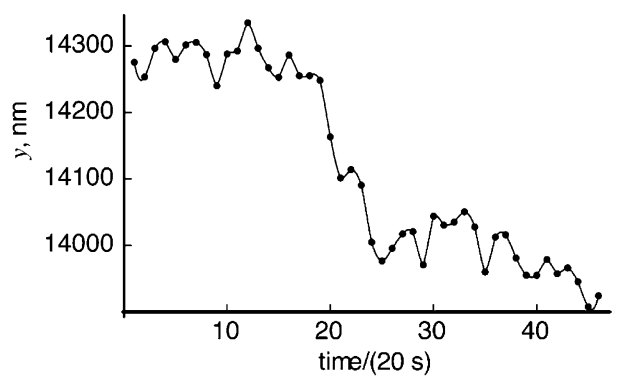

Figure 3. $y$-Component of a typical drift function computed for a set of six tethers with a length of $3477 \mathrm{bp}$. The horizontal axis indexes are consecutive, 20 -s time intervals consisting of 500 frames. The vertical axis is the $y$ position in nanometers. The solid line is an interpolation function chosen to pass through every point.

of the region as the intensity-weighted average of the contiguous pixels. (5) Repeat steps $1-4$ for the minimum region of the DIC image. (6) Average the two centers. To estimate the accuracy of the tracking, numerically created sequences with randomly positioned beads were evaluated with this algorithm. The error was less than one-third of a pixel, which is about 20 nm.

This procedure yielded data sequences consisting of $x y$ pairs, representing the locations of each of the four to six beads at successive time steps spanning about $20 \mathrm{~min}$. $x(t)$ and $y(t)$ for each bead were graphed and inspected visually (Figure 2 ). In many cases, a bead's movement abruptly ceased for some minutes. Presumably, this occurred when the bead temporarily stuck to the slide. However, in nearly every record, there were long intervals (over $360 \mathrm{~s}$ ) in which none of the beads showed such sticking. We cropped each data set to the longest such interval. In some cases, it was necessary to discard one of the beads altogether, to establish a long interval in which the remaining beads were well behaved. We discarded the entire time series if it contained fewer than four acceptable beads.

The observed positions of all of the beads in a given field of view appeared to drift slowly (see for example the $y$-component of Figure 2). To remove this drift from the data, we timeaveraged the center of mass of each group of four to six beads over 500 consecutive video frames $(20 \mathrm{~s})$ and then subtracted a vector interpolating function representing this motion (Figure 3) from the $x y$ pairs to obtain a drift-corrected time series. Taking the center of mass of several beads helped ensure that we removed the unwanted instrumental motion, common to all beads, while minimizing the amount of true diffusive motion inadvertently removed at the same time by this procedure. Appendix A discusses our choice of 500 frames for this filtering.

After drift correction, for most beads, the resulting excursions were symmetrically distributed around an anchor point (Figure 4). We subtracted the anchor coordinates from the corresponding time series to establish a series of projected displacement vectors for each tethered bead, $\rho_{\perp}(t)$.

We also found permanently stuck beads. Fourier transform of their time series showed no large peaks corresponding to 

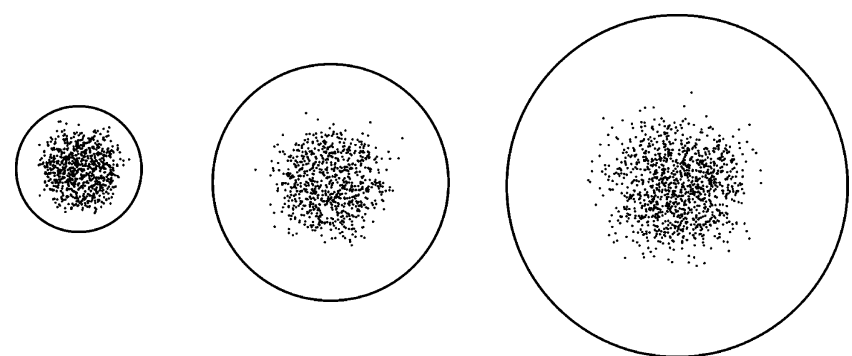

Figure 4. Left to right: Typical distributions of drift-corrected bead positions for tethers with a length of 957,2211 , and $3477 \mathrm{bp}$. The circles denote the geometric maximum deviation at each tether length, $\rho_{\max }=\left(L\left(L+2 R_{\text {bead }}\right)\right)^{1 / 2}=512,962$, and $1402 \mathrm{~nm}$, respectively. We found very few beads with multiple tethers, which are characterized by asymmetric motion about the anchor point on the slide. ${ }^{13}$ To confirm this, we evaluated the asymmetry of the "cloud" of bead positions as previously described. ${ }^{6}$ A statistical difference between the major and minor axes of the cloud is unavoidable, but beads for which it exceeded $10 \%$ ( $~ 5 \%$ of all beads analyzed) were discarded.

harmonic vibration in the apparatus. After implementing the above drift-correction procedure, there remained a residual motion of amplitude, $\sigma_{\text {stuck }} \approx \pm 15 \mathrm{~nm}$; see Appendix A. Because the beads were in fact stuck to the slide, this residual motion reflects instrumental noise (for example, high-frequency vibration or videotape jitter) not fully removed by our procedure. Because its amplitude adds roughly in quadrature with the true diffusive motion, which is always much larger than $15 \mathrm{~nm}$, we concluded that it had a minor effect on our results.

\section{Observed Brownian Motion}

For each time series (each observed bead), we then computed the average excursion, $\left\langle\rho_{\perp}\right\rangle$, and the complete probability distribution function, $P\left(\rho_{\perp}\right)$. In principle, each sequence could have a different distribution, $P\left(\rho_{\perp}\right)$, due to differences among beads, nonspecific sticking, the topography of the slide, and so forth. For each length of tethering DNA used, we actually found that most of the beads had very similar $P\left(\rho_{\perp}\right)$ 's, although a minority had significantly different distributions from the others. The minority generally corresponded to tethers that were effectively shorter than the majority (Figure 5).

The observed, mean excursion of the bead, $\left\langle\rho_{\perp}\right\rangle$, as a function of DNA tether length, $L$, is shown in Figure 6a. Each dot represents the measurement for one bead, and most of the data are closely grouped for each length of DNA, with only a few, well separated beads exhibiting smaller average excursions (red dots).

\section{Physical Model}

A mathematical model was developed to quantitatively simulate a tethered particle experiment. Although it was developed independently, it is essentially identical to that described by Segall et al. ${ }^{15}$ We employed the wormlike chain (WLC) model, which accurately accounts for many of the conformational properties of DNA, at least for molecules longer than about 200 base pairs. ${ }^{19}$ The biotin and digoxigenin linkages were treated as freely flexible pivots, at each end of a DNA molecule, and the bead and glass slide were represented as rigid, impenetrable bodies.

The following paragraphs explain how we calculated the statistical properties of the observable quantity (bead center projected on the xy plane) in terms of just three parameters (bead radius, $R_{\text {bead }}$, tether length, $L$, and persistence length, $\xi$ ). In our experiment, $R_{\text {bead }}$ equaled $240 \mathrm{~nm}$ and $L$ was determined for each construct as the total length in base pairs multiplied by $0.34 \mathrm{~nm} / \mathrm{bp}$. Thus, we tested the model by checking whether it could fit our experimental calibration curve, that is, whether any single choice of $\xi$ fit all our data at various $L$ values. In principle, we could have introduced the parameter $\sigma_{\text {stuck }}$ (see above), adding a random time series with this variance to our simulated data. In practice, we found that this correction had little effect on the results reported below.

The absence of any external stretching force on the DNA made the simulation of our system very easy. We evaluated our partition sums by a Monte Carlo integration method. We chose the discretization $l=5 \mathrm{bp}$ and attributed to each link an orientation, or orthonormal triad of vectors, $\left\{\mathbf{e}_{a}, a=1,2,3\right\}$. The third of these unit vectors represented the direction of the molecular backbone at that link (Figure 1). Starting with a randomly chosen initial orientation, $\left\{\mathbf{e}_{a}(1)\right\}$, with $\mathbf{e}_{3}(1)$ in the upper half-space, we chose the next link's orientation to be $\left\{\mathbf{e}_{a^{-}}\right.$ (2) $\}=\left\{\sum_{b} \mathbf{T}_{a b} \mathbf{e}_{b}(1)\right\}$, where again $a=1,2,3$. In this formula, $\mathbf{T}$ is a random rotation matrix obtained by exponentiating a generator matrix representing the rates at which the first triad must be rotated in order to become the second triad. In the wormlike chain model, these rates have a Gaussian distribution centered on zero, reflecting the assumption that the elastic energy

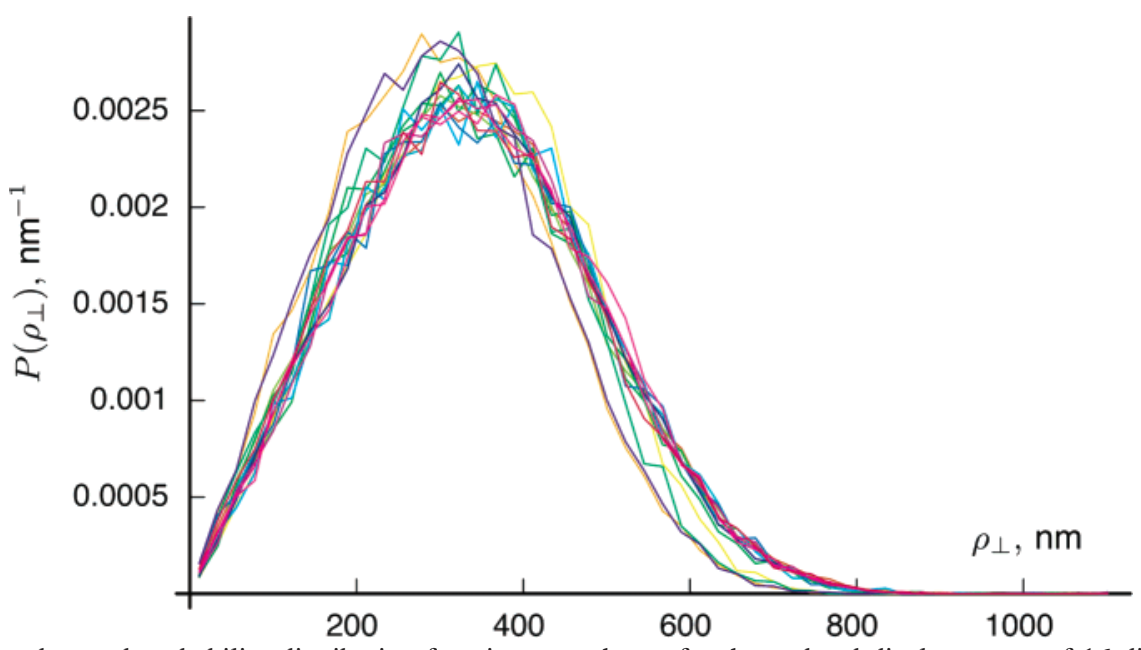

Figure 5. Experimentally observed probability distribution functions are shown for the $x y$ bead displacements of 16 different tethers, all with a length of $3477 \mathrm{bp}$. Twelve of the 16 curves shown are mutually similar. The remaining four, however, have both significantly shorter mean and maximum displacements. Such discrepant, minority, records were excluded from the fitting process. Nevertheless, they are shown as red dots in Figure 6. 

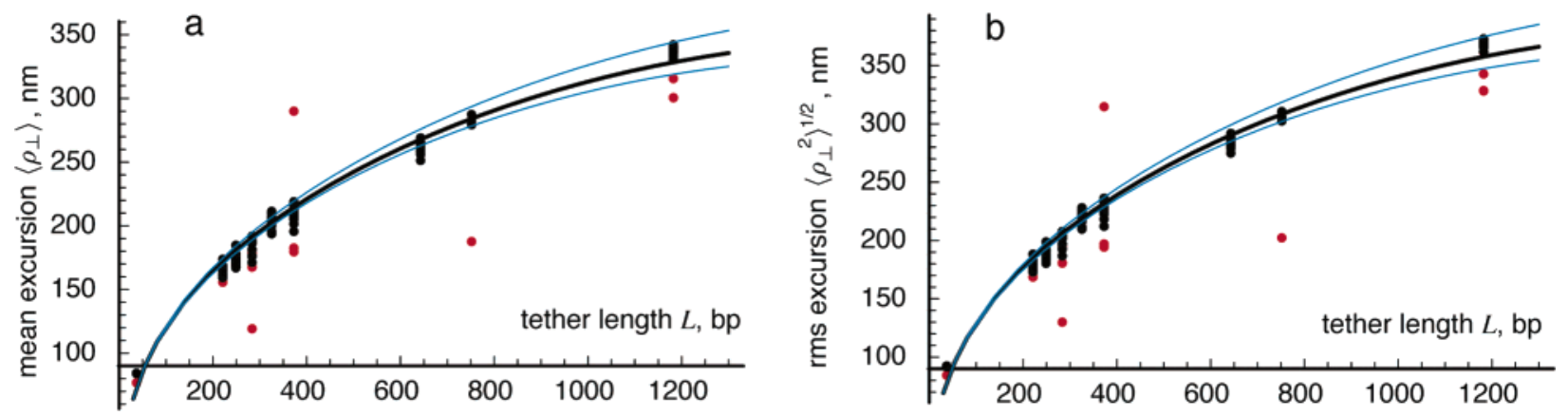

Figure 6. Observed excursion of the bead, as a function of DNA tether length, L: (a) mean excursion; (b) root-mean-square excursion. Each dot represents the amplitude of motion of one bead/tether, projected on the plane perpendicular to the optical axis. Black dots met all of our criteria (see text). Red dots met the visual criteria but had unusual probability distributions (Figure 5), or the associated bead excursions were severely anisotropic. The black curve shows a theoretical prediction taking $R_{\text {bead }}=240 \mathrm{~nm}$ and the best-fit persistence length $\xi=43 \mathrm{~nm}$ (see text, section 5). The top blue curve shows the same function with $\xi=48 \mathrm{~nm}$ and the bottom curve with $40 \mathrm{~nm}$.

is a harmonic (quadratic) function of bend and twist angles. The variance of this Gaussian was chosen to obtain the desired value of $\xi$. Subsequent links were obtained from their predecessors in the same way. The last vector, $\mathbf{m}$, describing the bead orientation, was chosen randomly in the half-space determined by the tangent to the last link, $\mathbf{e}_{3}(N)$. Finally, the position of the bead center was obtained by summing all of the tangent vectors, $\mathbf{e}_{3}(i)$, multiplying by the link length, and then adding the vector $R_{\text {bead }} \mathbf{m}$.

Configurations with steric clashes between the DNA, the bead, and the slide were discarded. (Chain-chain clashes do not significantly affect chain statistics for the range of lengths, $L$, studied in this work.) The algorithm ran rapidly because the fraction of chains discarded in this way was not large. Also, since each chain was generated independently of the others, we did not need the long pre-equilibration step required by Metropolis algorithms.

To facilitate fitting the model to the data, we computed the mean excursion radius, $\left\langle\rho_{\perp}\right\rangle$, for one fixed value, $\xi_{0}$, and a grid of different values of $R_{\text {bead }}$ and $L$ in the range of interest to us, and we summarized the result by using an interpolating function (Appendix B). Dimensional analysis shows that $\left\langle\rho_{\perp}\right\rangle / \xi$ can be written as a dimensionless function of $L / \xi$ and $R_{\text {bead }} / \xi$, so this calculation suffices to find $\left\langle\rho_{\perp}\right\rangle$ at any $\xi, L$, and $R_{\text {bead }}$ (Appendix B). Similar remarks apply for the root-mean-square excursion.

We then tabulated $L$ and the experimentally determined $\left\langle\rho_{\perp}\right\rangle$ for all of the DNA constructs we studied and attempted to choose a single $\xi$ value to best fit the theoretical distribution to them all. This fit (Figure 6a, black curve), with $R_{\text {bead }}$ equal to $240 \mathrm{~nm}$ (manufacturer's specification), is satisfactory and yields a DNA persistence length, $\xi$, equal to $43 \mathrm{~nm}$. We chose to fit the mean excursion (not the root-mean-square excursion) because it is less sensitive than the rms excursion to possible outlier points in the data. The figure shows that values outside the range $40 \mathrm{~nm}<\xi<48 \mathrm{~nm}$ gave unacceptable fits. Figure $6 \mathrm{~b}$ shows that the same fit value of $\xi$ also fit the rms excursion data.

\section{Comparison to Earlier Work}

Yin et al. also made a calibration curve for the TPM technique, based on experiments with DNA molecules of different lengths, labeled with $230 \mathrm{~nm}$ diameter beads. ${ }^{8}$ To compare their results to ours, we first converted their excursion parameter, $\delta$, to the root-mean-square projected excursion, $\sigma$ $=\left(\left\langle\rho_{\perp}{ }^{2}\right\rangle\right)^{1 / 2}$, reported in our simulations. Yin et al. averaged the video frames for about $4 \mathrm{~s}$; in the resulting images, moving beads appeared blurred, and their apparent diameter, $S$, was
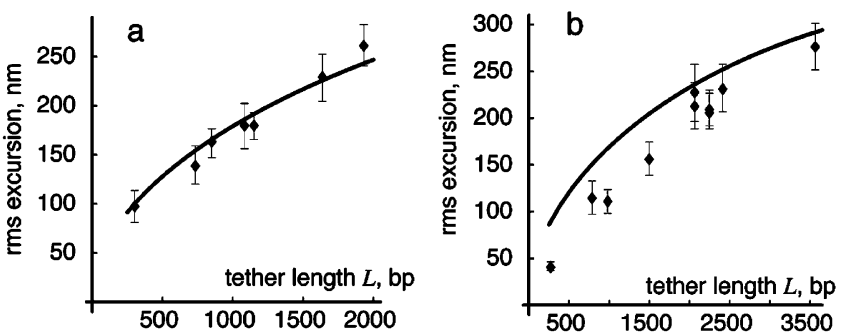

Figure 7. (a) Comparison of results from Yin et al. ${ }^{8}$ and our theoretical model, for $R_{\text {bead }}=115 \mathrm{~nm}$ and $\xi=43 \mathrm{~nm}$. The values of $\sigma$ were converted from Figure 3 of Yin et al. ${ }^{8}$ using eq 2 with $S_{0}=190 \mathrm{~nm}$. (b) Comparison of results from Pouget et al..$^{13}$ and our theoretical model, for $R_{\text {bead }}=100 \mathrm{~nm}$ and $\xi=43 \mathrm{~nm}$. We could not fit these data with any reasonable choice for $\xi$.

increased. Then, they evaluated $S$ by fitting with an equation of the form

$$
\begin{aligned}
& I(x, y)=A+B\left\{\exp \left(-\frac{x^{2}+(y-v)^{2}}{S^{2}}\right)-\right. \\
& \left.\exp \left(-\frac{x^{2}+(y+v)^{2}}{S^{2}}\right)\right\}
\end{aligned}
$$

Here $A, B, v$, and $S$ are fit parameters. Yin et al. represented the amplitude of motion of the bead with the parameter $\delta=S$ $-S_{0}$, where $S_{0}$ is the apparent size of a fixed bead. Although in our own measurements we prefer to work with the first moment of the excursion, our simulation can report any moment, and there is a straightforward conversion between the parameter $\delta$ and the second moment, $\sigma$, that allows us to compare our results to theirs. To find the relation, we note that the overall distribution of light intensity observed for a moving particle is the convolution of the intensity of a stationary particle and the probability distribution of excursions. This overall distribution of intensity thus has a mean-square deviation equal to the sum of the contributing factors: $S^{2}=S_{0}^{2}+\sigma^{2}$, or

$$
\sigma=\sqrt{\left(S_{0}+\delta\right)^{2}-S_{0}^{2}}
$$

Therefore, to extract $\sigma$ from $\delta$, we had to know $S_{0}$. We evaluated $S_{0}$ as follows. We numerically generated a light path profile of a bead, convolved it with a nominal point spread function of the microscope, and fit the resulting image with eq 1. Substituting the resulting $S_{0}$ value, $190 \mathrm{~nm}$, in eq 2 yielded the $\sigma$ values in Figure 7a, corresponding to the values of $\delta$ reported by Yin et al. Since they used different sized beads, we compared their data to the average excursions predicted with our model for $230 \mathrm{~nm}$ diameter beads tethered by DNA with a 


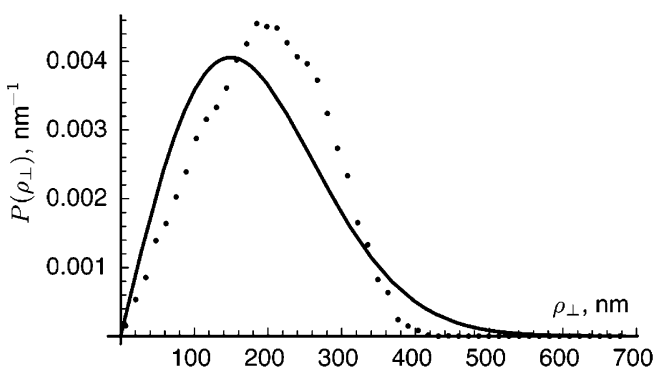

Figure 8. Typical observed probability distribution function, $P\left(\rho_{\perp}\right)$, for a $1096 \mathrm{bp}$ long tether (dots) that is distinct from that of a twodimensional Gaussian with the same mean-square deviation (curve). That is, the solid curve is the function $\left(2 \rho_{\perp} / \sigma^{2}\right) \exp \left(-\left(\rho_{\perp} / \sigma\right)^{2}\right)$, where $\sigma=211 \mathrm{~nm}$.

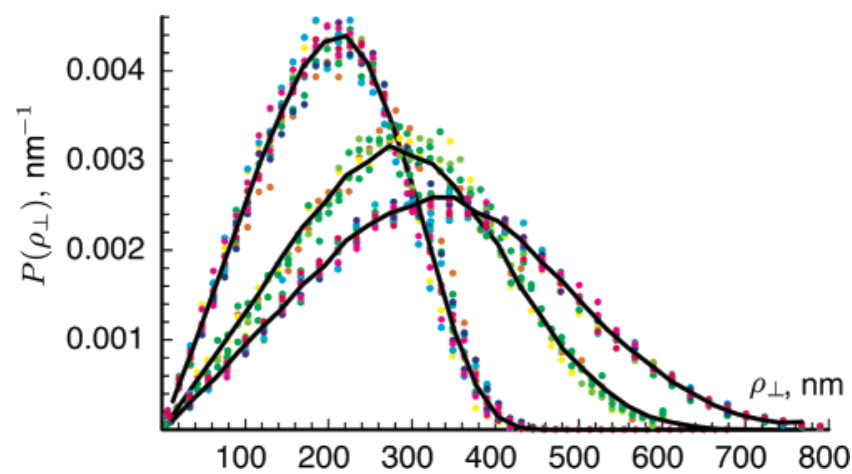

Figure 9. Probability distributions (histograms), $P\left(\rho_{\perp}\right)$, for tethers of length $L=957,2211$, and 3477 bp (left to right). Dots: For each length, distributions from several tethers are represented in different colors. Curves: Predictions from the model with $R_{\text {bead }}=240 \mathrm{~nm}$ and $\xi=43 \mathrm{~nm}$. The curves are not fits; they follow from the theory after choosing the single parameter $\xi$, as determined in Figure 6a. To make the curves, we generated about 700,000 chains at each of the indicated lengths and then made histograms of the chains' $\rho_{\perp}$ values.

persistence length of $43 \mathrm{~nm}$. Their data lie quite close to our theoretical curve.

Pouget et al. presented a significantly different calibration curve. ${ }^{13}$ They used particle tracking and evaluated the parameter $A_{\text {eq }}$, the standard deviation of the motion along one axis. To compare our results to those of Pouget et al., we noted that they expressed their results in terms of standard deviations of one coordinate, $x$ (equivalent to $y$ ). Thus, the $\sigma$ values in Figure $7 \mathrm{~b}$ are their reported standard deviations multiplied by $(2)^{1 / 2}$. Their experimental results are still roughly $30 \%$ lower than those predicted by our theoretical model, which is puzzling. The buffer they used contained $10 \mathrm{mM} \mathrm{Mg}^{2+}$, which should reduce the persistence length somewhat, ${ }^{20}$ but even reducing the assumed persistence length does not bring our model's prediction into accord with their data.

\section{Bead Position Distributions}

Perhaps the most convincing evidence of the adequacy of our model is that it correctly predicted the probability distributions for bead excursions. Experimentally measured probability distributions of the bead excursions for DNA of a given length are far from Gaussian, a fact previously noted by Pouget et al. ${ }^{13}$ (Figure 8).

Once we fit the single parameter $\xi$ to the calibration curve in Figure $6 \mathrm{a}$, the full probability distribution function, $P\left(\rho_{\perp} ; \xi\right.$, $L, R_{\text {bead }}$ ), for projected anchor-to-bead-center distance (see Figure 1) was predicted from the Monte Carlo simulations described above (see also ref 15). The predicted and measured probability distributions coincide without any additional fitting, as shown in Figure 9. That is, the model correctly predicts the nontrivial
(non-Gaussian) probability distribution functions of bead excursion at multiple different tether lengths.

\section{Discussion}

The accord between our data and model is remarkably good, in view of the model's many idealizations. For example, we assumed an ideal flow microchamber and bead surfaces (we neglected roughness, stickiness, electrostatics, etc.) and simply accepted the manufacturer's specification of $R_{\text {bead }}$. Also, our algorithm and data analysis are based on the simple assumption that the boundary value for the chain is a perfectly flexible linkage at each end. To some extent, we can justify these assumptions. One aim of our preparation is to completely coat the glass surface such that biotin-BSA molecules are scattered throughout a continuous layer of $\alpha$-casein protein. This protein layer blankets crevices and surrounds sharp asperities in the glass to create a smooth surface. Furthermore, the electrostatic Debye length is short under our solution conditions. ${ }^{15}$

Our fitted range for $\xi, 40 \mathrm{~nm}<\xi<48 \mathrm{~nm}$, includes the 45 and $47 \mathrm{~nm}$ values independently found upon stretching DNA in a similar buffer using magnetic tweezers. ${ }^{21,22}$ Our results are also one of the few direct measurements of a spatial probability distribution for a semiflexible polymer. Figure 9 shows that our simple theoretical model accurately reproduces these experimentally measurable distributions.

Blumberg et al. measured the full three-dimensional motion of the tethered bead and analyzed their data with an ad hoc, harmonic, potential well approximation, without including the motion of the bead at the free end of the DNA. ${ }^{6}$ Perhaps the discrepancy they noted between their predicted and measured spring constants of the DNA chain could be resolved by adapting our model to predict the full 3-D motion (see Appendix C).

One advantage of the TPM technique is that no external stretching force is applied, which could alter conformational transitions of the DNA. Nevertheless, the presence of the bead slightly extends the average end-to-end distance of the anchored DNA molecule with respect to the value found in a free molecule. Segall et al. estimated the corresponding extensional force at height $z$ above the surface as about $k_{\mathrm{B}} T / z$, where $k_{\mathrm{B}} T=$ $4 \mathrm{pN} \mathrm{nm}$ is the thermal energy scale at room temperature. ${ }^{15}$ For example, at a height of $300 \mathrm{~nm}$, typical of many of the tethers we considered (see Appendix C), this force is only about $0.013 \mathrm{pN}$. We can now ask whether such a force may significantly perturb protein-induced loops in DNA tethers, since this has been one of the major applications of the TPM technique. Taking the example of the DNA loop induced by the gal repressor protein and using the relations given in Lia et al., ${ }^{23}$ one can calculate that $0.013 \mathrm{pN}$ would reduce the loop lifetime by less than $1 \%$.

Thus, the TPM method negligibly alters the DNA mechanochemistry and can serve to monitor conformational changes in real time (for example, loop formation). We have presented a mathematical model that correctly predicted the full nonGaussian probability distributions of bead excursions measured for several different tether lengths. The model's success gives us confidence in the experimental protocols. Moreover, a priori knowledge of the excursion distributions will be useful as a basis for maximum-likelihood analyses of time series containing spontaneous transitions between conformational states, for example, those involving proteins that modify the topology of DNA.

Acknowledgment. We thank A. Favalli, A. Franzini, G. Galli, and R. Guerra for assistance with experiments. We thank S. Adhya, V. Croquette, D. Chow, I. Kulic, D. Lewis, P. Purohit, R. Phillips, S. Blumberg, D. Segall, and P. Wiggins for many 

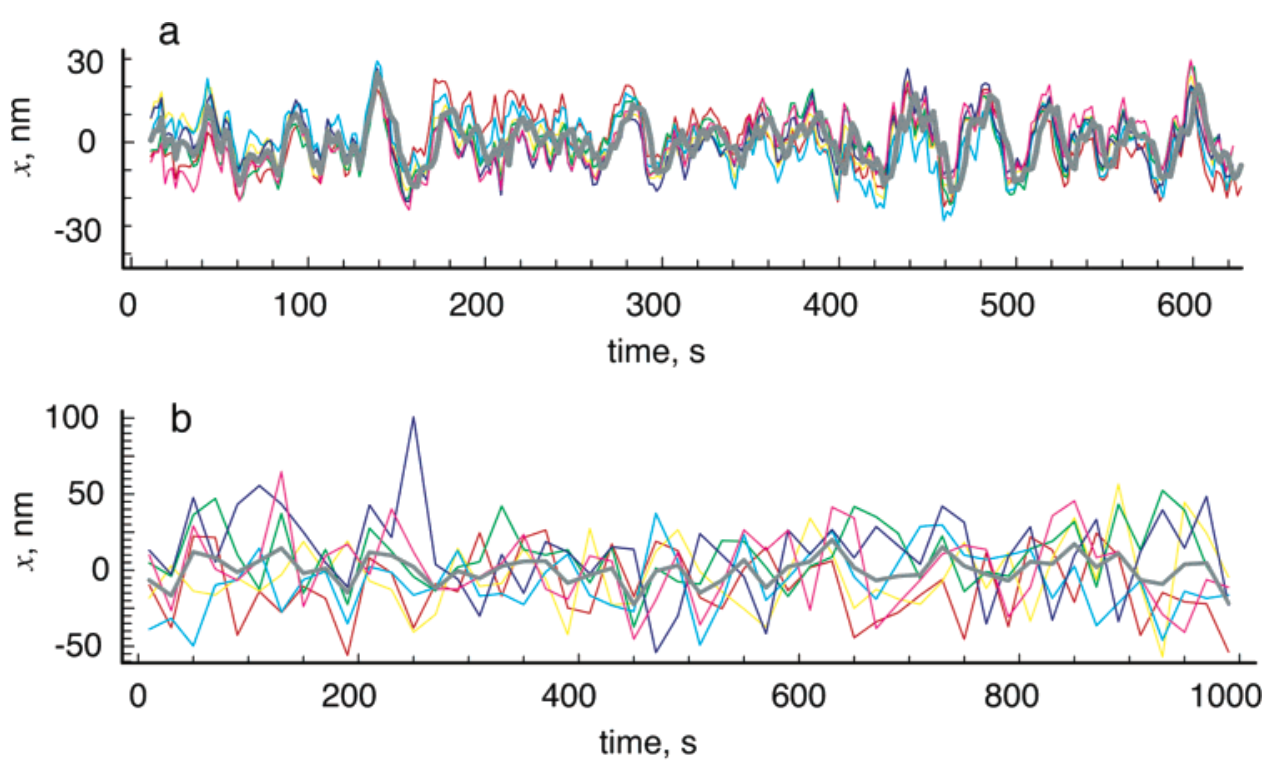

Figure 10. (a) Residual apparent motion of stuck beads for a typical data record. Time series of six bead positions ( $x$ coordinate only) after applying our drift correction with a window size of $M=20 \mathrm{~s}$. For easier visualization, we have further mean-filtered the data with a $2 \mathrm{~s}$ window. (b) Drift correction inferred by our procedure from simulated data with no true drift at all. The colored lines show six independently moving simulated beads, whose center of mass gives the gray line. The simulation assumed a tether length of $3477 \mathrm{bp}$.

useful discussions. This work was partially supported by the Human Frontier Science Program Organization (L.F. and P.C.N.), the Italian Ministry for Instruction, Universities and Research, COFIN 2002 and FIRB 2002 (L.F. and D.D.), and the National Science Foundation under grant DMR-0404674 and the NSF-funded NSEC on Molecular Function at the Nano/ Bio Interface DMR04-25780 (P.C.N.). L.F. and P.C.N. acknowledge the hospitality of the Kavli Institute for Theoretical Physics, supported in part by the National Science Foundation under grant PHY99-07949.

\section{Appendix A: Drift Subtraction Procedure}

The result of our real-time image processing algorithm is a time series of four to six $x y$ pairs representing the position of that number of simultaneously tracked beads. The recorded bead motion reflected a combination of several contributions. One component is the motion of interest to us, namely, independent Brownian motion of each bead relative to the surrounding fluid. Added to this is unwanted motion of our camera relative to the slide, which we may roughly partition into low-frequency ("drift") and a high-frequency ("vibration") components. Finally, there can be random error in the determination of each bead's center ("jitter"). We sought an optimal way to reduce the unwanted components of bead motion while preserving the interesting part. One way to do so could be to arrange for several fixed fiducial objects in each field of view, but our experimental setup did not provide this.

Instead, we noted that the largest unwanted motion was the slow drift evident in Figure 3. Applying a low-pass filter (moving average) to each time series allowed us to isolate this part of the motion. Accordingly, we averaged the vector bead positions over windows of duration $M$ (a quantity to be determined below), to obtain an inferred vector drift function. In addition, we reasoned that drift and vibration would be mainly translational, and hence common to all the beads in each field of view (we test this assumption below). Thus, we further averaged our drift function over all four to six beads tracked in each time series. The final drift function was interpolated to all times and then subtracted from all $x y$ pairs in the time series.

To make an optimal choice for the window size, $M$, we made a compromise. With too large a value of $M$ we would incorrectly

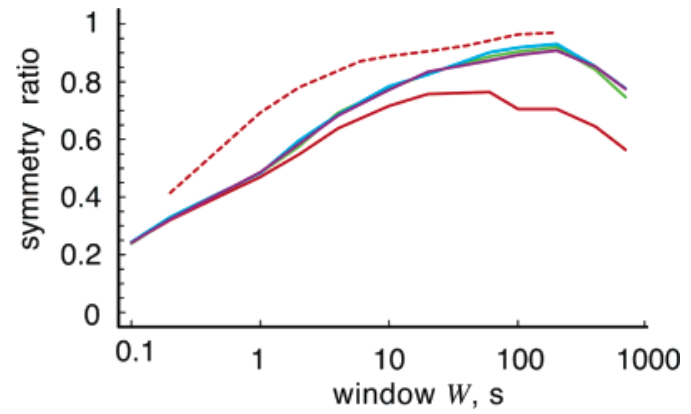

Figure 11. Solid lines: Average symmetry ratio for the Brownian motions of four typical beads on tethers with a length of $3477 \mathrm{bp}$, not corrected for drift, as a function of the window size, $W$ (see text). The solid red curve corresponds to an anomalous bead, of the sort rejected in the analysis of the main paper. Dashed line: For comparison, we selected points at random from the probability distribution appropriate for tethers of this length (Figure 9), grouped them into windows of various sizes, and again computed the average symmetry ratio. This quantity rises monotonically with increasing $W$, because of improved statistics; it does not fall at large $W$ because the points generated in this way have no drift.

interpret some genuine medium-frequency drift as Brownian motion. However, choosing too small a value of $M$ would have the opposite effect, inadvertently removing some true Brownian motion along with the drift. To make the best choice of $M$, we studied some auxiliary time series. We now explain this procedure and how it yielded $M=20 \mathrm{~s}$.

Stuck Bead Analysis. We prepared samples in which beads were immobilized on the slide by drying. The corresponding time series consisted purely of instrumental vibration, and drift, and tracking error. We computed our drift-correction function as usual and plotted the results (Figure 10a). The figure shows that our procedure eliminated the long-term drift but left an unwanted, residual apparent motion with an amplitude of about $\pm 15 \mathrm{~nm}$. Note also that indeed the bead motions roughly track each other, implying that most of the motion is really translational mechanical motion.

Simulated Motion Analysis. We performed a simple Brownian dynamics simulation of beads on tethers, to generate a data 

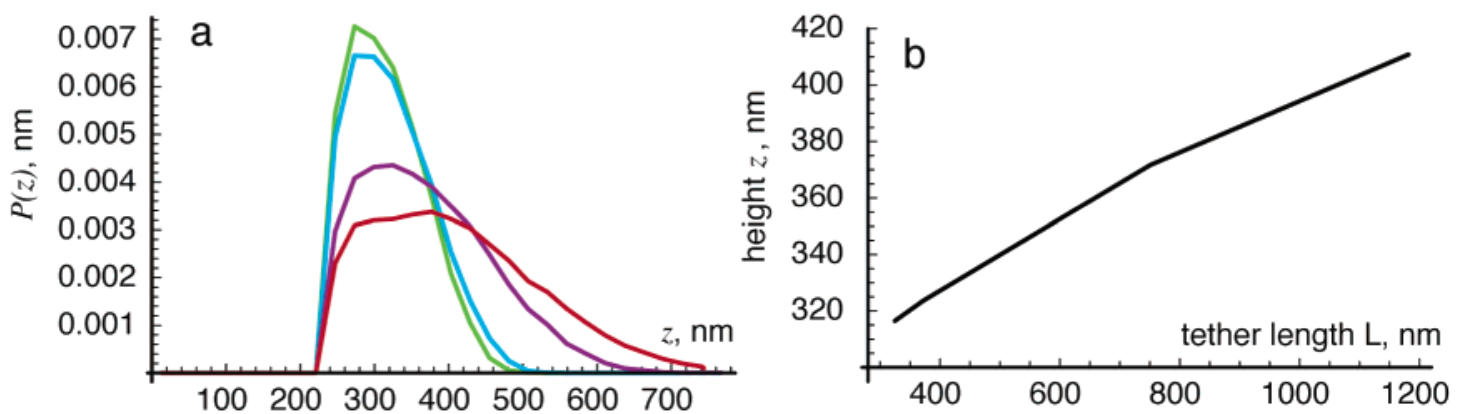

Figure 12. (a) Left to right: Predicted probability distribution functions for bead height, computed in our model for a bead with a radius of 240 $\mathrm{nm}$ with tether lengths of $957,1096,2211$, and $3477 \mathrm{bp}$. (b) The corresponding plot of mean height $\langle z\rangle$.

set with no drift or vibration whatsoever. Details of this simulation will appear elsewhere. We again computed our driftcorrection function on the simulated data using our usual procedure (Figure 10b). The figure shows that our procedure unavoidably interprets some true Brownian motion as drift, but the amplitude of this error, again about $\pm 15 \mathrm{~nm}$, is small.

Thus, decreasing the value of $M$ would improve the first of the errors discussed above but worsen the second, and vice versa for increasing it. We chose $M=20$ s to keep both contributions similar in magnitude.

Ellipticity Analysis. As a further check, we next analyzed the ellipticity of short stretches of uncorrected bead motion. That is, we considered windows of consecutive points, of width $W$, and found the vector mean and the (co)variances of $x x, x y$, and $y y$ in each window. From this, we found the eigenvalues of the covariance matrix and defined a symmetry parameter as their ratio. Figure 11 shows this symmetry ratio, averaged over all windows, as a function of window size, $W$.

We reasoned that a singly tethered bead would have a true Brownian motion that was circularly symmetric about the attachment point (symmetry ratio equal to unity). Nevertheless, for small values of $W$, the symmetry ratio should begin small (a handful of points is likely to be very anisotropic) and then rise as the statistics improve. However, at large $W$, instrumental drift will begin to stretch the apparent distribution, and the symmetry ratio should begin to fall again. Thus, the optimal window size, $M$, for drift correction should be somewhere near the peak of the symmetry ratio curve. This is a rough criterion, because different data runs had quite different low-frequency drift functions, but the figure shows that $M=20 \mathrm{~s}$ is again a reasonable choice.

Figure 11 illustrates two additional points applicable to tethered particle analysis. First, the ellipticity analysis can be used to screen for anomalous beads (solid red line at $\sim 0.4$ ). Second, the figure implies that it takes a relatively long time (tens of seconds) for a diffusing tethered bead to explore enough of the available phase space to appear symmetric.

Other Values. Finally, we repeated our entire analysis with a different window size of $M=8 \mathrm{~s}$ (data not shown), obtaining essentially the same results for the fit persistence length as in the main text.

\section{Appendix B: Calibration Curves from Monte Carlo Simulation}

Before fitting our model to data, we needed a convenient summary of its results, an interpolating function giving the model's prediction of the mean projected bead excursion in terms of the polymer persistence length, $\xi$, the bead radius, $R_{\text {bead }}$, and the contour length, $L$. Because these are the only length scales in the problem, we can simplify by noting that the result must have the form $\left\langle\rho_{\perp}\right\rangle=\left(\xi / \xi_{0}\right) F_{\text {mean }}\left(\left(\xi_{0} / \xi\right) R_{\text {bead }},\left(\xi_{0} / \xi\right) L\right)$, where the scaling function, $F_{\text {mean }}\left(R_{*}, L_{*}\right)$ is the extension evaluated at one particular value, $\xi_{0}$. We chose $\xi_{0}=43.4 \mathrm{~nm}$ and evaluated our Monte Carlo simulation in the range $190 \mathrm{~nm}$ $<R_{*}<340 \mathrm{~nm}, 0 \mathrm{~nm}<L_{*}<1200 \mathrm{~nm}$. We fit the result to a suitable interpolating function, finding that

$$
\begin{array}{r}
F_{\text {mean }}\left(R_{*}, L_{*}\right)=280-208 L_{*}{ }^{1 / 4}+48.4 L_{*}{ }^{1 / 2}-0.506 L_{*}+ \\
0.0000576 L_{*}{ }^{2}-3.21 R_{*}+2.44 L_{*}{ }^{1 / 4} R_{*}-0.480 L_{*}{ }^{1 / 2} R_{*}+ \\
0.00636 L_{*} R_{*}-1.081 \times 10^{-6} L_{*}{ }^{2} R_{*}+0.00672 R_{*}{ }^{2}- \\
0.00515 L_{*}{ }^{1 / 4} R_{*}{ }^{2}+0.001082 L_{*}{ }^{1 / 2} R_{*}{ }^{2}-0.00001493 L_{*} R_{*}{ }^{2}+ \\
2.60 \times 10^{-9} L_{*}{ }^{2} R_{*}{ }^{2}
\end{array}
$$

yielded a good fit. In this formula, all lengths are in nanometers.

For reference, we followed the same procedure for the rootmean-square excursion, obtaining

$$
\begin{gathered}
F_{\mathrm{rms}}\left(R_{*}, L_{*}\right)=264-199 L_{*}{ }^{1 / 4}+47.6 L_{*}{ }^{1 / 2}-0.506 L_{*}+ \\
0.0000680 L_{*}{ }^{2}-2.88 R_{*}+2.194 L_{*}{ }^{1 / 4} R_{*}-0.431 L_{*}{ }^{1 / 2} R_{*}+ \\
0.00587 L_{*} R_{*}-1.094 \times 10^{-6} L_{*}{ }^{2} R_{*}+0.00584 R_{*}{ }^{2}- \\
0.00449 L_{*}{ }^{1 / 4} R_{*}{ }^{2}+0.000949 L_{*}{ }^{1 / 2} R_{*}{ }^{2}-0.00001339 L_{*} R_{*}{ }^{2}+ \\
2.50 \times 10^{-9} L_{*}{ }^{2} R_{*}{ }^{2}
\end{gathered}
$$

\section{Appendix C: Height Distribution}

Although our experiments did not measure the height, $z$, of the bead from the surface, others' methods do yield this information. ${ }^{6}$ Accordingly, in Figure 12, we quote the results of our simulation for the mean height and for the full distribution of height values at various tether lengths.

\section{References and Notes}

(1) Bockelmann, U. Curr. Opin. Struct. Biol. 2004, 14, 368.

(2) Molloy, J. E.; Padgett, M. J. Contemp. Phys. 2002, 43, 241.

(3) Charvin, G.; Strick, T. R.; Bensimon, D.; Croquette, V. Annu. Rev. Biophys. Biomol. Struct. 2005, 34, 201.

(4) Gosse, C.; Croquette, V. Biophys. J. 2002, 82, 3314.

(5) Dixit, S.; Singh-Zocchi, M.; Hanne, J.; Zocchi, G. Phys. Rev. Lett. 2005, 94, 118101

(6) Blumberg, S.; Gajraj, A.; Pennington, M. W.; Meiners, J. C. Biophys. J. 2005, 89, 1272.

(7) Schafer, D. A.; Gelles, J.; Sheetz, M. P.; Landick, R. Nature 1991, $352,444$.

(8) Yin, H.; Landick, R.; Gelles, J. Biophys. J. 1994, 67, 2468

(9) Dohoney, K. M.; Gelles, J. Nature 2001, 409, 370.

(10) Finzi, L.; Gelles, J. Science 1995, 267, 378.

(11) Gelles, J.; Yin, H.; Finzi, L.; Wong, O. K.; Landick, R. Biophys J. 1995, 68, 73S. 
(12) Tolic-Norrelykke, S. F.; Engh, A. M.; Landick, R.; Gelles, J. J. Biol. Chem. 2004, 279, 3292.

(13) Pouget, N.; Dennis, C.; Turlan, C.; Grigoriev, M.; Chandler, M.; Salome, L. Nucleic Acids Res. 2004, 32, e73.

(14) Qian, H.; Elson, E. L. Biophys. J. 1999, 76, 1598

(15) Segall, D. E.; Nelson, P. C.; Phillips, R. Phys. Rev. Lett. 2006, 96, 088306

(16) Finzi, L.; Dunlap, D. Methods Enzymol. 2003, 370, 369.

(17) Zurla, C.; Franzini, A.; Galli, G.; Dunlap, D. D.; Lewis, D. E. A.; Adhya, S.; Finzi, L. J. Phys.: Condens. Matter 2006, 18, S225.

(18) Allemand, J. F. Micromanipulation d'une molécule individuelle d'ADN. Ph.D. Thesis, Université Pierre et Marie Curie Paris VI, 1997.
(19) Wiggins, P. A.; Nelson, P. C. Phys. Rev. E: Stat., Nonlinear, Soft Matter Phys. 2006, 73, 031906.

(20) Baumann, C. G.; Smith, S. B.; Bloomfield, V. A.; Bustamante, C. Proc. Natl. Acad. Sci. U.S.A. 1997, 94, 6185.

(21) Strick, T. R.; Croquette, V.; Bensimon, D. Proc. Natl. Acad. Sci. U.S.A. 1998, 95, 10579

(22) Wang, M. D.; Yin, H.; Landick, R.; Gelles, J.; Block, S. M. Biophys. J. 1997, 72, 1335 .

(23) Lia, G.; Bensimon, D.; Croquette, V.; Allemand, J. F.; Dunlap, D.; Lewis, D. E.; Adhya, S.; Finzi, L. Proc. Natl. Acad. Sci. U.S.A. 2003, 100,11373 